\title{
Cadernos Cemarx
}

2019

Centro de Estudos Marxistas 


\section{FICHA CATALOGRÁFICA ELABORADA PELA BIBLIOTECA DO IFCH - UNICAMP}

Bibliotecário: Paulo Roberto de Oliveira - CRB 8/6272

Cademos CEMARX / publicação do Centro de Estudos Marxistas do Instituto de Filos ofia e Ciências Humanas da Universidade Estadual de Campinas. n. 1 (2004 -) Campinas: UNICAMP/IFCH, 2004-

2019 (12)

ISSN - 1980-2099 (versão impressa)

ISSN - 2318-065x (versão on-line)

1. Marxismo. 2. Ciência Política. 3. Nicos Poulantzas. 4. Teoria do Estado 5. Socialismo.

I. Instituto de Filosofia e Ciências Humanas. Universidade Estadual de Campinas. II. Título.

CDD - 320.532 


\section{CADERNOS CEMARX}

\section{COMITÊ EDITORIAL}

Breno Augusto de Oliveira Santos

Felipe de Queiroz Braga

Mariana Shinohara Roncato

Murillo van der Laan

Nátaly Santiago Guilmo

Nicolle Garcia Berti

Octávio Fonseca Del Passo

Rodolfo Soarez Moimaz

\section{Revisores}

Cláudia Vanessa Bergamini

Gabrielle Champi Duarte

\section{Conselho editorial}

Adriano Codato (UFPR), Alexander Maximilian Hilsenbeck Filho (CLACSO Argentina), Ana Elisa Corrêa (UFRJ), Andréia Galvão (Unicamp), Armando Boito Jr. (Unicamp), Caio Navarro de Toledo (Unicamp), Caio Bugiato (UFRRJ), Daniela Vieira dos Santos (Unicamp) Danilo Martuscelli (UFFS), Davisson Cangussu de Souza (Unifesp), Demian Melo (UFFS), Eduardo Martins Ráo (Unicamp), Elaine Amorim (Unicamp), Elaine Bezerra (Unicamp), Eliel Machado (UEL), Ellen Gallerani Corrêa (Unicamp), Fábio Durão (Unicamp), Fabio Mascaro Querido (Unicamp), Francisco Pereira Farias (UFPI), Gonzalo Rojas (UFCG), Gualtiero Marini (Unicamp), Gustavo Casasanta Firmino (Unicamp), Hector Benoit (Unicamp), Hélio Ázara de Oliveira (UFCG), Henrique Amorim (Unifesp), Henrique Tahan Novaes (Unesp), Isabel Loureiro (Unicamp), Isabela Meucci (USP), Jair Pinheiro (Unesp), João Campinho (Unicamp), João Pedro de Toledo Camargo Hadler (UFRJ), João Quartim de Moraes (Unicamp), Jorge Grespan (USP), Jose Marcos Novelli (Ufscar), Julia Gomes Souza (Unicamp), Leandro de Oliveira Galastri (Unesp), Lígia Osório (Unicamp), Lívia Moraes (UFES), Luciana Aliaga (UFPB), Luciana Henrique da Silva (Unicamp), Luciano Martorano (UFAL), Lúcio Flávio Rodrigues de Almeida (PUC/SP), Maíra Machado Bichir (UNILA) Márcio Bilharinho Naves (Unicamp), Marcos Cassin (USP), Marcos Del Roio (Unesp), Maria Orlanda Pinassi (Unesp), Nair Gadelha (USP), Nathalia Oliveira (Unicamp), Nelson Prado Alves (Unicamp), Patrícia Rocha Lemos (Unicamp), Patrícia Vieira Trópia (UFU), Paula Marcelino (USP), Renato Perissinotto (UFPR), Rafael Dias Toitio (Unicamp) Rodolfo Palazzo Dias (UFSC), Rodrigo Passos (Unesp), Ruy Braga (USP), Sabrina Areco (UEMS), Santiane Arias (Unifesp), Sávio Cavalcante (Unicamp), Selma Venco (Unicamp), Sérgio Lessa (UFAL), Tatiana Berringer (UFABC), Tatiana Prado Vargas (Unicamp), Thiago Barison (USP), Vinícius Oliveira Santos (Unicamp), Virgínia Fontes (UFF), Zaira Vieira (Unimontes). 


\author{
Cadernos Cemarx \\ Universidade Estadual de Campinas - Unicamp \\ Instituto de Filosofia e Ciências Humanas - IFCH \\ Cemarx - Centro de Estudos Marxistas \\ Cidade Universitária "Zeferino Vaz" \\ Campinas - SP I Brasil \\ www.unicamp.br/cemarx \\ Fone 0xx19 3521.1639
}

Editoração, finalização miolo e capa: Setor de Publicações/IFCH

Capa original: Alexandre Linhares Guedes - Professor do Departamento de Artes (DARTES) da Universidade Federal Rural do Rio de Janeiro (UFRRJ)

"A laranja é uma alegoria do país!

Por fora, a falsa aparência de que tudo vai bem e de que a democracia, mesmo aviltada, é a capa perfeita para implementação de um regime ditatorial velado. Na face interna não há o que se esconder, pois o Estado-nação brasileiro está carcomido pela submissão aos norte-americanos, ao capital internacional e as elites de rapina que subtraem o Brasil desde sua descoberta.

A imagem também faz referência as relações espúrias entre o atual governo e o crime organizado". 


\section{Sumário}

\section{CADERNOS CEMARX}

DOSSIÊ - O pensamento de Nicos Poulantzas: reflexões sobre o Estado e as classes sociais

Apresentação

Bobbio crítico de Poulantzas

Armando Boito Júnior

As condições de existência das classes sociais

no capitalismo

Francisco Pereira de Farias

Nicos Poulantzas e a Questão do Estado:

uma análise fundamentada na obra

Poder Político e Classes Sociais

Greice dos Reis Santos

51

Estrutura e Autonomia Relativa:

conceitos complementares

Jair Pinheiro

O desenvolvimento do conceito

poulantziano de hegemonia

Octávio Fonseca Del Passo

Nicos Poulantzas, a reprodução capitalista e a luta de classes Angela Lazagna 
Dualidade do poder, estratégia do comunismo

e definhamento do Estado.

O debate entre Althusser e Poulantzas

Fabio Bruschi

Observações sobre o totalitarismo

Nicos Poulantzas

Sobre o impacto popular do fascismo

Nicos Poulantzas

Entrevista com Nicos Poulantzas:

o Estado e a transição ao socialismo

Nicos Poulantzas 